BULLETIN OF THE

AMERICAN MATHEMATICAL SOCIETY

Volume 80, Number 4, July 1974

\title{
THREE STRUCTURE THEOREMS IN SEVERAL COMPLEX VARIABLES
}

\author{
BY REESE HARVEY
}

The purpose of this article is to describe three recent structure theorems in the theory of several complex variables and to point out a few of the many applications of these three theorems. In the first section we discuss a characterization of those currents (defined on an open subset of $\boldsymbol{C}^{n}$ ) which correspond to integration over complex subvarieties. The second section is concerned with the structure of positive, $d$-closed currents. Finally, in the third section, a characterization of boundaries of complex subvarieties of $\boldsymbol{C}^{n}$ is discussed. A common thread in the techniques of proof involves "potential theory" for several complex variables.

1. Recognizing currents that correspond to integration over complex subvarieties. Suppose $V$ is a complex subvariety of an open set in $C^{n}$ with each irreducible component of $V$ of dimension $k$. It is sometimes useful to consider, instead of the point set $V$, the linear functional "integration over $V$ ", which we denote by $[V]$. More precisely, for each compactly supported smooth form $\varphi$ of degree $2 k$, define $[V](\varphi)$ by integrating $\varphi$ over the manifold points of $V$. A basic fact about complex subvarieties is that in a neighborhood of a singular point the $2 k$-volume of the manifold points is finite (see [4], [16], or [24]). Therefore $[V](\varphi)$ is locally estimated by a constant times the supremum of the coefficients of the form $\varphi$. This implies that $[V]$ is a current (of real dimension $2 k$ or (real) degree $2 n-2 k$ ). In fact, this estimate implies that the current $[V]$ viewed as a differential form with distribution coefficients actually has measures for coefficients.

There are several ways of recognizing which currents are of the form $[V]$ where $V$ is a complex subvariety. The most elementary result of this kind says that if $V$ is a real $2 k$ dimensional submanifold of $\boldsymbol{C}^{n} \cong \boldsymbol{R}^{2 n}$ and at each point of $V$ the tangent space to $V$, considered as a real linear subspace of $\boldsymbol{R}^{2 n} \cong \boldsymbol{C}^{n}$, is in fact a complex linear subspace, then $V$ is a complex submanifold. It will shed light on later results to reinterpret this elementary result as follows. Suppose $u$ is a current of degree $2(n-k)($ dimension $2 k)$

An expanded version of an invited address delivered to the Society at the 78th Summer Meeting in Missoula, Montana, 1973; received by the editors December 14, 1973. Research partially supported by a Sloan Fellowship and NSF GP-19011.

AMS (MOS) subject classifications (1970). Primary 32-02, 32C25; Secondary 49F20, 37F05. 
corresponding to integration over a smooth $2 k$ dimensional oriented submanifold $V$ of an open set in $\boldsymbol{R}^{2 n} \cong \boldsymbol{C}^{n}$. Then $V$ is a complex submanifold if and only if $u$ is of bidegree $n-k, n-k$ (or bidimension $k, k$ ); where a current $u$ is said to be of bidegree $n-k, n-k$ (bidimension $k, k$ ) if $u(\varphi)=0$ for all $\varphi=f d z^{I} \wedge d \bar{z}^{J}=f d z_{i_{1}} \wedge \cdots \wedge d z_{i_{p}} \wedge d \bar{z}_{j_{2}} \wedge \cdots \wedge d \bar{z}_{j_{q}}$ except when $p=q=k$ (where $f$ is a smooth compactly supported function). The proof of the above fact easily reduces to the trivial case where $V$ is a real linear subspace of $\boldsymbol{R}^{2 n} \cong C^{n}$.

Another way of recognizing currents corresponding to integration over subvarieties is provided by a special case of a real-variable result of Federer (see [4, 4.1.15 p. 373]). This result implies that if a current of real dimension $2 k$ defined on an open subset of $C^{n}$ is, closed under exterior differentiation, supported on an irreducible complex subvariety $V$, and of a special type called locally flat, then the current is a constant multiple of [V] (see King [14, Proposition 3.1.3]). For an example of an application of this result suppose that $f$ is a holomorphic function. Then $\log |f|$ is plurisubharmonic (and hence locally integrable) and in fact pluriharmonic outside $V=\{z: f(z)=0\}$ (see [12] or [18] for a discussion of plurisubharmonic functions). A pluriharmonic function is a function which is annihilated by all of the operators $\partial^{2} / \partial z_{i} \partial \bar{z}_{j}$. Therefore $(i / \pi) \partial \bar{\partial} \log |f|$ is a current supported on $V$. Using the result mentioned above it is easy to see that:

$(i / \pi) \partial \bar{\partial} \log |f|$ is the current $\sum m_{j}\left[V_{j}\right]$ where $\left\{V_{j}\right\}$ is the family of irreducible components of $V$ and each $m_{j} \in Z^{+}$is the multiplicity of $f$ vanishing on $V_{j}$.

This formula is of fundamental importance in several complex variables (for example in Nevanlinna theory and residue theory).

Next we examine special properties of currents of the form $[V]$ (where $V$ is a complex subvariety), or more generally of integral linear combinations. Suppose $\left\{V_{j}\right\}$ is a sequence of irreducible subvarieties of dimension $k$, which satisfy the condition that only a finite number of them intersect any given compact set, and that $\left\{m_{j}\right\}$ is a sequence of integers. Then the sum $\sum m_{j}\left[V_{j}\right]$ is called a holomorphic $k$-chain. (If $k=n-1$ this notion is equivalent to the classical notion of a divisor.) If, in addition, each $m_{j}$ is positive then $\sum m_{j}\left[V_{j}\right]$ is called a positive holomorphic $k$-chain.

Now we list some of the properties of a holomorphic $k$-chain $u=$ $\sum m_{j}\left[V_{j}\right]$.

(1) $u$ is of bidimension $k, k$ (bidegree $n-k, n-k$ ).

(2) $u$ is $d$-closed.

Here $d$ denotes exterior differentiation. This condition can be interpreted geometrically as saying that each $V_{j}$ has no boundary, and is rigorously deduced from the fact that $d u$ must be supported in the singular 
points of the $V_{j}$ (i.e., a set of real dimension $\leqq 2 k-2$ ) which is too small to support a $2 k-1$ dimensional boundary (see Federer [4] for the details).

(3) $u$ has measure coefficients and the $2 k$-density $\Theta_{2 k}(u, z)$ is a positive integer at each point $z$ in the support of $u$.

Here $\Theta_{2 k}(u, z) \equiv \lim _{r \rightarrow 0^{+}} M_{B(z, r)}(u) / c_{2 k}{ }^{2 k}$, where: $c_{2 k}$ is the volume of the unit ball in $\boldsymbol{R}^{2 k}, B(z, r)$ is ball about $z$ of radius $r$, and $M_{\Omega}(u) \equiv$ $\sup \left\{|u(\varphi)|: \varphi\right.$ is a smooth $2 k$ form with compact support in $\Omega$ and $\left.\|\varphi\|_{\infty} \leqq 1\right\}$ denotes the $2 k$-volume or mass of $u$ on $\Omega$. For a proof that the above limit exists see Lelong [18]. For example, if $V=\left\{z \in C^{2}: z_{1}^{2}-z_{2}^{3}=0\right\}$ and $u=[V]$ then $\Theta_{2}(u, z)$, the density of $u$ at $z$, is equal to zero on $C^{2}-V$, one on the manifold points $V-\{0\}$, and two at $z=0$. The integer $\Theta_{2 k}([V], z)$ is, in fact, always the multiplicity of $V$ at $z$ (Draper [3]).

StruCtURe TheORem I. Given a current $u$ on an open subset of $C^{n}$ which satisfies (1), (2), and (3) above then $u$ is a holomorphic k-chain.

See Harvey and Shiffman [11] for the proof. Various results of geometric measure theory are employed in this proof. One of the important steps (assume $k=n-1$ ) is to construct a meromorphic function $f$ with $(i / \pi) \partial \bar{\partial} \log |f|=u$.

If $u=\sum n_{j}\left[V_{j}\right]$ is a positive holomorphic $k$-chain, then in addition to properties (1), (2), and (3) above, the following holds:

(4) $u$ is positive.

By definition this means that for each smooth compactly supported function $\varphi \geqq 0$ and for each choice of linear coordinates $z=\left(z_{1}, \cdots, z_{n}\right)$, the quantity $u\left(\varphi(i / 2) d z_{1} \wedge d \bar{z}_{1} \wedge \cdots \wedge(i / 2) d z_{k} \wedge d \bar{z}_{k}\right)$ is greater than or equal to zero.

COROLLARY 1.1. Under the hypothesis of Theorem I if u also satisfies (4) then $u$ is a positive holomorphic k-chain.

This very important special case of Theorem I was conjectured by Lelong [17] and is due to King [14].

One of the most interesting applications of Theorem I, which is not also a consequence of the Corollary, is a theorem of Lawson and Simons [15]. They prove that every stable current on complex projective $n$-space is a holomorphic chain. See [11] for other applications. (In particular, note the uniqueness result, Theorem 3.6, for a special class of Plateau problems in $C^{n}$.)

A current $u$ of the form $\sum c_{j}\left[V_{j}\right]$ with $\left\{V_{j}\right\}$ as above and each $c_{j}$ a positive real number is called a positive holomorphic $k$-chain with real coefficients. These currents can be characterized as follows (see Harvey and King [8]).

THEOREM 1.2. Suppose $u$ is a current defined on an open subset of $\boldsymbol{C}^{n}$ which is positive, of bidimension $k, k, d$-closed, and $\Theta_{2 k}(u, z)$ is bounded 
below by a positive constant on each compact subset of the support of $u$. Then $u$ is a positive holomorphic $k$-chain with real coefficients.

Although this theorem naturally belongs in this section, its proof (entirely unlike that of Theorem I) depends on a fundamental result of Bombieri [1] and [2] which is the basis for structure Theorem II of the next section.

2. Density points of positive, $d$-closed currents of bidimension $k, k$. The currents which satisfy: (1) bidimension $k, k$ (bidegree $n-k, n-k$ ), (2) $d$-closed, and (4) positive, need not correspond to integration over subvarieties. For example $(i / 2) \partial \bar{\partial}|z|^{2}=\sum_{j=1}^{n}(i / 2) d z_{j} \wedge d \bar{z}_{j}, i \partial \bar{\partial} \log \left(1+|z|^{2}\right)$, and $i \partial \bar{\partial} \log |z|^{2}(n>1)$, are $d$-closed, positive currents of bidegree 1,1 (bidimension $n-1, n-1$ ) which do not correspond to integration over subvarieties. More generally if $\varphi$ is any plurisubharmonic function then $i \partial \bar{\partial} \varphi$ is a positive current (which is also $d$-closed and of bidegree 1,1 ). In fact this can be taken as the definition of a plurisubharmonic function. Suppose $\varphi$ is a distribution and

$$
i \partial \bar{\partial} \varphi=\sum \frac{\partial^{2} \varphi}{\partial z_{j} \partial \bar{z}_{k}} i d z_{j} \wedge d \bar{z}_{k}
$$

is positive. (For 1,1 currents $u=\sum u_{j k} i d z_{j} \wedge d \bar{z}_{k}$ is positive if and only if $\sum u_{j k} \lambda_{j} \bar{\lambda}_{k}$ is a positive measure for each $\lambda \in C^{n}$.) One can prove that $\varphi$ is locally integrable and that if one defines $\varphi$ pointwise by $\tilde{\varphi}(z)=$ ess $\lim _{w \rightarrow z} \varphi(w)$, then $\tilde{\varphi}$ is classically plurisubharmonic. See Lelong [18] or Vladimirov [25] for a full discussion.

Locally every $d$-closed positive current of bidegree 1,1 arises as in the above discussion. That is, given such a current $u$ on the ball $B(0, r)$ in $C^{n}$ there exists a solution $\varphi$ to $i \partial \bar{\partial} \varphi=u$ (see [11] for example).

The present section is concerned with the points of high concentration, or density, of a positive current $u$. Interestingly, for positive, $d$-closed currents $u$,

$$
\Theta_{2 k}(u, z)=\lim _{r \rightarrow 0^{+}} \frac{M_{B(z, r)}(u)}{c_{2 k} r^{2 k}}
$$

not only exists, but is the limit of a function which is decreasing as $r$ decreases (see Lelong [18, Proposition 10], and Federer [4, Theorems 5.4.3 and 5.4.19]). Consider the example $u=(i / \pi) \partial \delta \log |z|$ mentioned above. Then $\Theta_{2 n-2}(u, z)=0$ for $z \in C^{n}-\{0\}$ and $\Theta_{2 n-2}(u, 0)=1$.

The next structure theorem is due to Siu [22]. This result was conjectured by Harvey and King [8]. 
Structure Theorem II. Suppose $u$ is a positive, d-closed current of bidimension $k, k$ (bidegree $n-k, n-k$ ) on an open subset of $C^{n}$. Then for each $c>0, E_{c}=\left\{z: \Theta_{2 k}(u, z) \geqq c\right\}$ is a complex subvariety of dimension $\leqq k$.

This structure theorem depends on a fundamental result of Bombieri [1] and [2].

STRUCTURE THEOREM II'. Suppose $\varphi$ is a plurisubharmonic function on the ball $B(0, r)$ in $C^{n}$. Then for each $c>0$ the set $I_{c}=\left\{z \in B(0, r): e^{-\varphi / c}\right.$ is not integrable in any neighborhood of $z\}$ is a proper subvariety of $B(0, r)$.

Bombieri [2] also made the following estimates.

THEOREM 2.1. Suppose $\varphi$ is given as in the above theorem and let $u=$ $(i / \pi) \partial \bar{\partial} \varphi$ on $B(0, r)$. Then $E_{2 n c} \subset I_{c} \subset E_{\gamma c}$ for each $c>0$, where $\gamma$ is a constant depending on $n$.

These results are used in [8] to prove Theorem 1.2 of the last section.

Remark. Skoda [23, Proposition 7.1] has shown that $\gamma$ can be chosen equal to 2. This result, $I_{c} \subset E_{2 c}$, is sharp since for $\varphi=\log \left|z_{1}\right|^{2}$ and $u=$ $(i / \pi) \partial \bar{\partial} \varphi=2\left[\left(z_{2}, \cdots, z_{n}\right)\right.$ hyperplane $], e^{-\varphi}=\left|z_{1}\right|^{-2}$ is not integrable near the origin while $u$ has density 2 at the origin.

Skoda [23] obtained the following very important generalization of Theorem 2.1 from positive currents of bidimension $n-1, n-1$ to positive currents of general bidimension.

THEOREM 2.2. Suppose $u$ is a positive, $d$-closed current of bidimension $k, k$ (bidegree $p, p$ where $p+k=n$ ) defined near the origin in $C^{n}$. Then there exists a plurisubharmonic function $\varphi$ such that $E_{c}(u) \subset I_{1}(\varphi) \subset E_{p c / n}(u)$.

In other words, $E_{c}(u)$ is contained in a subvariety $I_{1}(\varphi)$ which is not much bigger than $E_{c}(u)$ in the sense that $I_{1}(\varphi)$ is contained in $E_{p n / c}(u)$.

Siu's proof of Structure Theorem II proceeds from the above results. Let me illustrate one of the ideas of the proof by considering the following special case. Suppose $u$ is a positive $d$-closed current of bidegree 1,1 (bidimension 1, 1) defined in a neighborhood of the origin in $C^{2}$ and assume $c>0$ is given. By Theorem $\mathrm{II}^{\prime}$ and Theorem 2.1 above, $E_{c}$ is contained in a complex curve $V$ near the origin. For the sake of simplicity assume that $V$ is a connected complex manifold. If $E_{c}=V$ the proof is complete, so assume that $E_{c} \subsetneq V$, or equivalently that $c^{\prime}=\inf \left\{\Theta_{2}(u, z): z \in V\right\}$ is $\langle c$. First consider the case where $c^{\prime}=0$. By Theorem 2.1 and the Remark we have $E_{c} \subset I_{c / 4} \subset E_{c / 2}$ Choose $z \in V$ with $\Theta_{2}(u, z)<c / 2$. Then $z \notin E_{c / 2}$ and hence $z \notin I_{c / 4}$. Therefore $V \cap I_{c / 4}$ is a complex subvariety of dimension zero which contains $E_{c}$. This proves that if $c^{\prime}=0$ then $E_{c}$ is a finite point set (i.e., a zero dimensional subvariety). Finally, assume $c^{\prime}>0$ and let $v=$ $u-c^{\prime}[V]$. Suppose for the moment we have shown that $v$ is positive. Then 
$\Theta_{2}(u, z) \geqq c$ implies that $\Theta_{2}(v, z)=\Theta_{2}(u, z)-c^{\prime} \geqq c-c^{\prime}$, that is $E_{c}(u) \subset$ $E_{c-c^{\prime}}(v)$. By the argument given above applied to $v, E_{c-c^{\prime}}(v)$ is a zero dimensional subvariety. Therefore, if $E_{c} \subsetneq V$ then $E_{c}$ is a zero dimensional subvariety. To complete the proof we must show that $u-c^{\prime}[V]$ is positive. This is a result of Siu of independent interest which holds in greater generality.

Proposition 2.3. Suppose $u$ is a d-closed positive current of bidimension $k, k$, and that $V$ is a pure $k$ dimensional subvariety with irreducible components $\left\{V_{\imath}\right\}$. Let $c_{i}=\inf \left\{\Theta_{2 k}(u, z): z \in V_{i}\right\}$. Then $u-\sum c_{i}\left[V_{i}\right]$ is positive.

A sketch of the proof is given for $k=n-1$ (the case needed above). For simplicity assume $V$ is irreducible. The general case is reduced to this case. Define a measure $\mu$ by $\mu(\Omega)=M_{\Omega}(u)(\mu$ is the volume measure of $u)$. Let $\sigma$ denote $2 n-2$ dimensional volume measure induced by $V$ (i.e., $\sigma(\Omega)=M_{\Omega}([V])$, which is the same as the $2 n-2$ Hausdorff measure of $\Omega \cap V)$. Since $\Theta_{2 n-2}(u, z) \geqq c$ on $V$, a generalization of the Lebesgue differentiation theorem (Federer $[4,2.10 .19(3)]$ ) says that $\mu-c \sigma$ is a positive measure on $V$ (and hence a positive measure). Next solve $i \partial \bar{\partial} \varphi=u-c[V]$. Then $\varphi$ is plurisubharmonic outside $V$. One can show that $\frac{1}{4} \Delta \varphi=\mu-c \sigma$ (see [18] or [4] for example). Since $\mu-c \sigma$ is a positive measure $\varphi$ must be subharmonic. Therefore $\varphi$ is locally bounded above. Since $\varphi$ is plurisubharmonic outside $V$ and locally bounded above across $V$, it follows that $\varphi$ is plurisubharmonic (see, for example, Harvey [6, part c of the theorem on p. 132]). Therefore $u-c[V]=i \partial \bar{\partial} \varphi$ is positive.

By using both Proposition 2.3 and Structure Theorem II a strengthened version of Theorem 1.2 can be obtained.

TheOREM 2.4. Suppose $u$ is a positive, $d$-closed, current of bidegree $(n-k, n-k)$ (bidimension $k, k)$ on an open subset of $C^{n}$. There exist irreducible subvarieties $\left\{V_{j}\right\}$ and positive constants $c_{j}$ such that $u=\sum c_{j}\left[V_{j}\right]+v$ where $\sum c_{j} M_{\Omega}\left(\left[V_{j}\right]\right)<\infty$ for each relatively compact $\Omega$ and where $v$ is positive with the complex varieties $E_{c}(v)$ of dimension $\leqq k-1$. The above representation is unique.

Proof. Let $c_{1}=\sup \left\{c: \operatorname{dim}_{C} E_{c}=k\right\}$ and let $V_{1}$ denote the union of the components of $E_{c_{1}}$ of dimension $k$. By definition $E_{c_{1}}=\bigcap_{c<c_{1}} E_{c}$, which implies that $E_{c_{1}}=E_{c}$ for some $c<c_{1}$ (see, for example, Narasimham [19, Theorem 2, p. 70]). This proves that $E_{c_{1}}$ is of dimension $k$, or that $V_{1} \neq \varnothing$, unless $c_{1}=0$. By Proposition 2.3, $u_{1} \equiv u-c_{1}\left[V_{1}\right]$ is positive. Let $c_{2}=$ $\sup \left\{c: \operatorname{dim}_{C} E_{c}\left(u_{1}\right)=k\right\}$ and let $V_{2}$ denote the union of the components of $E_{c_{2}}\left(u_{1}\right)$ of dimension $k$. As above, either $c_{2}=0$, in which case the proof is complete, or $u_{2} \equiv u-c_{1}\left[V_{1}\right]-c_{2}\left[V_{2}\right]$ is positive. Continuing we have $u_{N}=u-\sum_{j=1}^{N} c_{j}\left[V_{j}\right]$ is positive. One can easily show that the coefficients 
of the currents $u_{N}$ (which are measures) converge weakly in measure. Let $v$ denote the limit of the $u_{N}$. Then $v$ is positive, and $d$-closed. Also since

$$
M_{\Omega}\left(\sum_{1}^{N} c_{j}\left[V_{j}\right]\right)=M_{\Omega}\left(u-u_{N}\right)=M_{\Omega}(u)-M_{\Omega}\left(u_{N}\right) \leqq M_{\Omega}(u)<\infty,
$$

$M_{\Omega}\left(u_{N}-v\right)=M_{\Omega}\left(\sum_{N+1}^{\infty} c_{j}\left[V_{j}\right]\right)$ converges to zero; that is, $u_{N}$ converges to $v$ is the mass norm.

Bombieri's original use of a global version of Theorem II' in "algebraic values of meromorphic maps" [1] provides a fascinating application of the results in this section. Siu uses his results in this section to prove an extension theorem for meromorphic maps conjectured by Griffiths [5]. (See Siu [21] for the full strength of the result and the proof.)

THEOREM 2.5. Suppose A is a subvariety of a complex manifold $X$ of a codimension $\geqq 2$, and that $Y$ is a compact Kähler manifold. Every meromorphic map $f$ from $X-A$ to $Y$ extends to a meromorphic map $f$ from $X$ to $Y$. (Cf. Griffiths [5], Shiffman [20], and Harvey [7].)

3. Recognizing boundaries of complex varieties. First suppose that a compact oriented, $2 k-1$ dimensional smooth submanifold $M$ of $C^{n}$ is the boundary of a complex $k$ dimensional manifold $V$. Then $T_{z}(V)=$ $T_{z}(M) \oplus[v]$ where $[v]$ is the real line spanned by the normal to $M$ (with respect to $V$ ) at $z$. Therefore, the orthogonal complement in the complex vector space $T_{z}(V)$ of the complex line spanned by $v$, is a complex vector space of complex dimension $k-1$ lying in $T_{z}(M)$. That is

$$
\operatorname{dim}_{C}\left(T_{z}(M) \cap i T_{z}(M)\right)=k-1 \quad \text { for all } z \in M .
$$

If this condition is satisfied $M$ will be called maximally complex. (Analogously with $\S 1$ one can show that the current $u=[M]$ is of bidimension $(k, k-1)$ union $(k-1, k)$ if and only if $M$ is maximally complex.)

Structure Theorem III. Let $M$ be a compact, orientable, $(2 k-1)$ dimensional real submanifold of $C^{n}$ of class $C^{r}$ with $k, r \geqq 2$. If $M$ is maximally complex, then $M$ is the boundary (as a current) of a uniquely determined bounded complex subvariety $V$ of $C^{n}-M$.

For details about boundary regularity and further results see the announcement [9]. Detailed proofs are to appear in Harvey and Lawson [10].

The analogue of Theorem III for $k=1$ (i.e., $M$ a real curve) is a known result. It is obtained by replacing the vacuous hypothesis that $M$ be a maximally complex real curve by the condition that $\int_{M} \omega=0$ for all holomorphic 1-forms on $\boldsymbol{C}^{n}$. The question of which one dimensional real curves bound complex curves of one dimension has received a lot of 
attention beginning with fundamental results of Wermer [26] dealing with real-analytic curves. (In most of the works the focus is on finding analytic structure in the spectrum of function algebras.)

Theorem III includes, as the special case where $M$ is the graph of a function defined on the boundary of an open set in $C^{n-1}$, Bochner's infinitesimal version of Hartogs' phenomenon (cf. Hörmander [12, Theorem 2.3.2' and Theorem 2.6.13]).

See Hunt and Wells [13] for some local extension results employing the connection made in [10] between extending manifolds and extending functions.

\section{REFERENCES}

1. E. Bombieri, Algebraic values of meromorphic maps, Invent. Math. 10 (1970), 267-287. MR 46 \#5328.

2. - Addendum to my paper "Algebraic values of meromorphic maps", Invent. Math. 11 (1970), 163-166.

3. R. N. Draper, Intersection theory in analytic geometry, Math. Ann. 180 (1969), 175-204. MR 40403.

4. H. Federer, Geometric measure theory, Die Grundlehren der math. Wissenschaften, Band 153, Springer-Verlag, New York, 1969. MR 41 \#1976.

5. P. A. Griffiths, Two theorems on extensions of holomorphic mappings, Invent. Math. 14 (1971), 27-62. MR 45 \#2202.

6. R. Harvey, Removable singularities and structure theorems for positive currents, Proc. Sympos. Pure Math., vol. 23, Amer. Math. Soc., Providence, R.I., 1973, pp. 129-133.

7. - - A result on extending positive currents, Amer. J. Math. (to appear).

8. R. Harvey and J. P. King, On the structure of positive currents, Invent. Math. 15 (1972), 47-52. MR 45 \#5409.

9. R. Harvey and B. Lawson, Boundaries of complex analytic varieties, Bull. Amer. Math. Soc. 80 (1974), 180-183.

10. - Boundaries of complex analytic varieties (in preparation).

11. R. Harvey and B. Shiffman, A Characterization of holomorphic chains, Ann. of Math. (to appear).

12. L. Hörmander, An introduction to complex analysis in several variables, Van Nostrand, Princeton, N.J., 1966, MR 34 \#2933.

13. L. R. Hunt and R. O. Wells, Jr., Holomorphic extension for non-generic C.R. submanifolds, Proc. Sympos. Pure Math., vol. 27, Amer. Math. Soc., Providence, R.I. (to appear).

14. J. King, The currents defined by analytic varieties, Acta Math. 127 (1971), 185-220.

15. H. B. Lawson and J. Simons, On stable currents and their application to global problems in real and complex geometry, Ann. of Math. 98 (1973), 427-450.

16. $\mathrm{P}$. Lelong, Intégration sur un ensemble analytique complexe, Bull. Soc. Math. France 85 (1957), 239-262. MR 20 \#2465.

17. - Propriétés métriques des ensembles analytiques complexes, Séminaire Lelong, 6e année: 1965/66, no. 2, Secrétariat mathématique, Paris, 1966. MR 36 \#4941.

18. - Fonctions plurisousharmoniques et formes différentielles positives, Gordon and Breach, New York (distributed by Dunod, Paris), 1968. MR 39 \#4436.

19. R. Narasimhan, Introduction to the theory of analytic spaces, Lecture Notes in Math., no. 25, Springer-Verlag, New York and Berlin, 1966. MR 36 \#428. 
20. B. Shiffman, Extension of positive line bundles and meromorphic maps, Invent. Math. 15 (1972), 332-347.

21. Y.-T. Siu, Analyticity of sets associated to Lelong numbers and the extension of meromorphic maps, Bull. Amer. Math. Soc. 79 (1973), 1200-1205.

22. — Analyticity of sets associated to Lelong numbers and the extension of closed positive currents (to appear).

23. H. Skoda, Sous-ensembles analytiques d'ordre fini ou infini dans $\boldsymbol{C}^{n}$, Bull. Soc. Math. France 100 (1972), 353-408.

24. G. Stolzenberg, Volumes, limits, and extensions of analytic varieties, Lecture Notes in Math., no. 19, Springer-Verlag, Berlin and New York, 1966. MR 34 \#6156.

25. V. S. Vladimirov, Methods of the theory of functions of several complex variables, "Nauka", Moscow, 1964; English transl., M.I.T. Press, Cambridge, Mass., 1966. MR 30 \#2163; 34 \#1551.

26. J. Wermer, The hull of a curve in $C^{n}$, Ann. of Math. (2) 68 (1958), 550-561. MR 20 \#6536.

27. H. B. Lawson, Minimal varieties in real and complex geometry, Montreal Lecture Notes.

Current address: Department of Mathematics, Rice University, Houston, Texas 77001 